\title{
Occurrence of Possible Rheumatologic Immune- Related Adverse Events (rh-irAEs) Associated with Immune Checkpoint Inhibitor (ICI) Therapy
}

\author{
Suzanne Anjohrin · Anna Sheahan · Robert Suruki · Jeffrey L. Stark • \\ Victor S. Sloan
}

Received: June 28, 2021 / Accepted: August 12, 2021 / Published online: September 7, 2021

(C) The Author(s) 2021

\section{ABSTRACT}

Introduction: Current epidemiologic literature of rheumatologic immune-related adverse events (rh-irAEs) consists of clinical trials, case reports, or smaller, single-center series. We evaluate the occurrence of rh-irAEs during immune checkpoint inhibitor (ICI) therapy from US commercial claims data.

Methods: Patients newly initiating ICI therapy in commercial claims data were eligible for inclusion. Rh-irAEs were defined using $\geq 1$ International Classification of Diseases (ICD)-9 or ICD-10-Clinical Modification (CM) claims for selected events, ranging from joint pain and myalgia to ankylosing spondylitis and psoriasis. The percentage of patients experiencing rhirAEs after ICI initiation was determined.

Results: A total of 5722 patients initiating an ICI between January 1, 2012, and June 30, 2018,

Supplementary Information The online version contains supplementary material available at https:// doi.org/10.1007/s40744-021-00359-z.

S. Anjohrin $(\bowtie) \cdot$ A. Sheahan · R. Suruki · J. L. Stark UCB Pharma, 1950 Lake Park Dr SE, Smyrna, GA 30080, USA

e-mail: suzanne.anjohrin@ucb.com

V. S. Sloan

The Peace Corps, Washington, DC, USA

V. S. Sloan

Rutgers Robert Wood Johnson Medical School, New

Brunswick, NJ, USA were included; 201 patients (3.5\%) had a history of rheumatic disease. Among the 5521 patients without a history of rheumatic disease, $29.6 \%$ experienced $\geq 1$ rh-irAE in follow-up, decreasing to $22.6 \%$ when assessing events for which there was no diagnostic history. Limiting to claims for rh-irAE with a rheumatologist provider, the proportion of patients experiencing an event decreased to $0.9 \%$. Among patients with a history of rheumatic disease, $71.6 \%$ experienced $\geq 1$ rh-irAE. Limiting to events for which the patient did not have a history during baseline, 35.3\% experienced an event.

Conclusions: Occurrence of rh-irAEs during ICI use is higher in patients with pre-existing rheumatic disease compared to those with no pre-existing rheumatic disease. However, the most common events were not definitive rheumatic diseases but rather symptoms, such as pain in joints. Occurrence of events associated with a rheumatologist provider was substantially lower, suggesting that either patients are not referred to a rheumatologist or referral does not result in confirmation of the diagnosis by the rheumatologist.

Keywords: Ankylosing spondylitis; Cohort study; Commercial claims; Immune checkpoint inhibitor; Psoriasis; Psoriatic arthritis; Rheumatoid arthritis; Rheumatologic immune-related adverse event; Rheumatologist 


\section{Key Summary Points}

Why carry out this study?

To date, epidemiologic literature of rheumatologic immune-related adverse events (rh-irAEs) consists of clinical trials, case reports, or smaller, single-center series.

This analysis seeks to determine the occurrence of rh-irAEs among patients undergoing immune checkpoint inhibitors (ICI) therapy using a large, geographically diverse commercial claims dataset.

What was learned from the study?

Over the 78 months evaluated, it was found that patients with a prior history of rheumatic disease had a higher occurrence of rh-irAEs; the most common rh-irAEs were not definitive rheumatic diseases but rather symptoms, such as pain in joints.

Awareness of the potential for these events is warranted, particularly among patients with a history of rheumatic disease, in order to support effective clinical management; for health care providers, such information can reinforce treatment vigilance and the provision of tailored, patient-oriented care.

\section{INTRODUCTION}

Immune checkpoint inhibitor (ICI) therapy revolutionized cancer treatment $[1,2]$ and was recognized with the 2018 Nobel Prize in Physiology or Medicine [3]. ICIs block inhibitory signals in a patient's immune system to enhance the host anti-tumor response [2, 4-7]. Despite the potentially dramatic benefit of ICIs against metastatic disease, this enhanced response generates a unique set of adverse events, collectively referred to as immune- related adverse events (irAEs). IrAEs can affect the dermatologic, gastrointestinal, hepatic, pancreatic, pulmonary, renal, endocrine, neurologic, hematologic, ophthalmologic, cardiac, and/or musculoskeletal systems. The irAE types most commonly reported are dermatologic, gastrointestinal, endocrine, and pulmonary [7-10].

All adverse events reported in oncology are graded according to their severity using the Common Terminology Criteria for Adverse Events (CTCAE) [5]. The CTCAE scale ranges from 1 (mild) to 5 (death). However, the application of grade-specific criteria may be subjective, and published analyses and clinical trials of irAEs frequently include only irAEs of $\geq$ grade 3 (severe or medically significant), or the results presented are restricted to irAEs that occurred in $\geq 5 \%$ of patients $[1,8,11]$. The combination of inconsistent reporting and the use of subjective criteria limits the synthesis of data across studies, particularly with regard to irAE subtypes. Differentiating incidence versus prevalence of irAEs is challenging, owing to variability in study-specific selection criteria and the specific irAE outcomes of interest.

IrAEs affect 53.8, 26.5, and $17.1 \%$ of patients undergoing anti-tumor therapy in clinical trials with anti-CTLA-4, anti-PD-1, and anti-PD-L1, respectively [12]. Fewer data are available regarding rheumatologic irAEs (rh-irAEs), which includes events that are also termed musculoskeletal. Diagnoses of rh-irAEs include, but are not limited to, all or a selection of the following conditions: arthralgias, myalgias, arthritis, tenosynovitis, polymyalgia rheumatica (PMR), sicca syndrome, and myositis $[5,13,14]$. RhirAEs are frequently deemed "less common" in the literature and variable estimates are reported due to the ambiguous definition of rh-irAEs, inconsistent selection criteria across studies, and irregular reporting of lower-grade irAEs $[5,7,8,14]$. Additionally, there is some evidence that rh-irAEs have a longer time to symptom onset than non-rheumatic irAEs, which could contribute to lower detection rates $[11,13]$.

To date, epidemiologic literature of rh-irAEs consists of clinical trials, case reports, or smaller, single-center series. This analysis seeks to 
determine the occurrence of rh-irAEs among patients undergoing ICI therapy using a large, geographically diverse commercial claims dataset.

\section{METHODS}

\section{Study Design}

A retrospective analysis of US IBM $^{\circledR}$ MarketScan $^{\circledR}$ Commercial Claims and Encounters with Medicare supplement data was conducted. The study population included patients newly initiating an ICI between January 1, 2012, and June 30, 2018. Claims were identified by the Healthcare Common Procedure Coding System (HCPCS) for ipilimumab, pembrolizumab, nivolumab, atezolizumab, avelumab, durvalumab, and cemiplimab. New initiation was defined as having no prior ICI claims in the preceding 6-month baseline period. The first ICI claim was the index claim, the date of which was the index date.

Formal ethical review boards were not consulted for this study. All analysis was performed via de-identified and anonymized database with permission of the data owner to be performed and presented at an aggregate level. The database is Health Insurance Portability and Accountability Act (HIPAA) compliant, and all patient data were de-identified before delivery. The study was conducted in accordance with the Helsinki Declaration of 1964, and its later amendments.

\section{Participants}

Patients were required to be 18 years or older at the index date and have a 6-month baseline and (at a minimum) 6-month follow-up period of continuous enrollment for medical and pharmacy coverage surrounding the index date. Gaps in coverage of up to 60 days in database enrollment were allowed. Patients were included in the analysis until there was a discontinuation of ICI therapy, defined as 6 months without claims or the end of database enrollment, whichever came first.

\section{Study Procedures and Evaluations}

Claims for outcomes of interest considered to be potential rh-irAEs during the period of ICI exposure and/or all available follow-up time were identified by occurrence of an International Classification of Diseases (ICD)-9 or ICD10-Clinical Modification (CM) code for one of the following events: "arthropathies, monoarthritis, polyarthritis", "myalgia, myositis, rheumatism", "psoriasis" (PSO), "systemic sclerosis", "sarcoidosis", "psoriatic arthritis" (PsA), "rheumatoid arthritis" (RA), "pain in joint", "ankylosing spondylitis" (AS), "systemic lupus erythematosus" (SLE), "giant cell arteritis", "polymyalgia rheumatica", "sicca syndrome", "polymyositis", "dermatomyositis, and "dermatopolymyositis". PSO was included as an event of interest due to its relationship with PsA. Refer to Table S1 in the electronic supplementary material for a comprehensive list of relevant ICD-9 and ICD-10-CM codes for consideration in identifying potential rh-irAEs.

All patients treated with ICIs were assessed for exposure to rheumatologists during baseline and follow-up periods. Patients were considered to have a pre-existing rheumatic condition if they had $\geq 1$ claim in baseline for a biologic for which there is a rheumatologic indication, or if they had $\geq 1$ claim for the defined events of interest, PSO, PsA, RA, or AS, in baseline. While some patients with a history of biologic use without a claim for PSO, PsA, RA, or AS would be defined in the analysis as having a pre-existing rheumatic condition, some of the biologics assessed also have non-rheumatologic indications (e.g., Crohn's disease, ulcerative colitis). A list of medications assessed with nonrheumatologic indications is provided in Table S2 of the electronic supplementary material.

\section{Statistical Analysis}

Descriptive analyses were conducted to examine the occurrence of rh-irAEs among patients undergoing ICI therapy, stratified by patient history of rheumatic disease in baseline. To examine new occurrences of rh-irAEs, 
frequencies of rh-irAEs unique to the follow-up period were described. Rh-irAEs diagnosed in baseline, other than PSO, PsA, RA, and AS, were excluded from these results due to insufficient patient numbers, and the analysis was performed in both strata. Since mild events are generally managed by the treating oncologist, rh-irAE severity was approximated by examining the proportion of events in which the provider type on the claim was a rheumatologist. Descriptive outcomes were presented as frequencies and proportions.

\section{RESULTS}

\section{Patient Disposition and Baseline Characteristics}

A total of 5722 patients initiated an ICI between January 1, 2012, and June 30, 2018, met inclusion criteria, and were included in the analysis; $5521 / 5722$ (96.5\%) patients did not have evidence of pre-existing rheumatic disease in baseline; 201/5722 (3.5\%) patients did have a history of pre-existing rheumatic disease.

More than half of patients without a history of rheumatic disease $(56.4 \%)$ were male, compared to $48.8 \%$ of patients with a history of rheumatic disease. The most frequently observed ICI was nivolumab monotherapy, accounting for $47.3 \%$ of patients without a history of rheumatic disease and $44.3 \%$ of patients with a history of rheumatic disease, followed by pembrolizumab monotherapy at $26.4 \%$ and $33.3 \%$, respectively. Mean treatment duration was 10.3 months for both patient cohorts.

\section{Outcomes}

\section{Patients Without a History of a Rheumatic Disease in Baseline}

Among patients without a history of pre-existing rheumatic disease $(n=5521), 29.6 \%$ $(n=1636)$ experienced $\geq 1$ rh-irAE (Table 1$)$. Pain in joints was the most common rh-irAE during the follow-up period $(24.5 \%, n=1354)$ (results not shown). The mean time to first claim for an rh-irAE from ICI initiation was 4.2 months. The proportion of patients with $\geq 1$ rh-irAE with rheumatologist provider for the claim was $0.9 \%(n=47$; Table 1$)$.

After limiting to rh-irAEs that were unique to the follow-up period, $22.6 \%(n=1247)$ of 5521 patients had $\geq 1$ new rh-irAE (Table 1). The most frequent event was pain in joints $(17.2 \%$, $n=952$ ), followed by myalgia, myositis, and rheumatism, collectively $(4.1 \%, \quad n=229$; Table 2). When further limiting to rh-irAEs only diagnosed in follow-up and where a rheumatologist was the provider type for the diagnostic claim, the proportion of patients experiencing

Table 1 Frequency and percentages of rh-irAEs in follow-up by patients' rheumatologic history in baseline

\begin{tabular}{|c|c|c|}
\hline & $\begin{array}{l}\text { Patients with a history of rheumatic } \\
\text { disease }(n=201) \\
\text { rh-irAE occurrence in follow-up }\end{array}$ & $\begin{array}{l}\text { Patients without a history of rheumatic } \\
\text { disease }(n=5521)\end{array}$ \\
\hline \multicolumn{3}{|l|}{ All rh-irAEs } \\
\hline rh-irAE, any provider & $144(71.6 \%)$ & $1636(29.6 \%)$ \\
\hline $\begin{array}{l}\text { rh-irAE, rheumatologist } \\
\text { provider }\end{array}$ & $42(20.9 \%)$ & $47(0.9 \%)$ \\
\hline \multicolumn{3}{|c|}{ Only rh-irAEs for which there was no diagnosis in baseline } \\
\hline rh-irAE, any provider & $71(35.3 \%)$ & $1247(22.6 \%)$ \\
\hline $\begin{array}{l}\text { rh-irAE, rheumatologist } \\
\text { provider }\end{array}$ & $10(5.0 \%)$ & $43(0.8 \%)$ \\
\hline
\end{tabular}

rh-irAE rheumatologic immune-related adverse event 
Table 2 Frequency and percentages of rh-irAE diagnoses in follow-up by patients' rheumatologic history in baseline, limiting to events undiagnosed at baseline

\begin{tabular}{|c|c|c|c|c|}
\hline & \multicolumn{2}{|c|}{$\begin{array}{l}\text { Patients with a history of rheumatic } \\
\text { disease }(n=201)\end{array}$} & \multicolumn{2}{|c|}{$\begin{array}{l}\text { Patients without a history of rheumatic } \\
\text { disease }(n=5521)\end{array}$} \\
\hline & Any provider & Rheumatologist & Any provider & Rheumatologist \\
\hline Any rh-irAE & $71(35.3 \%)$ & $10(5.0 \%)$ & $1247(22.6 \%)$ & $43(0.8 \%)$ \\
\hline AS & 0 & 0 & $4(0.1 \%)$ & 0 \\
\hline Arthropathies & $10(5.0 \%)$ & $2(1.0 \%)$ & $95(1.7 \%)$ & $6(0.1 \%)$ \\
\hline Dermatomyositis & 0 & 0 & $1(0.0 \%)$ & 0 \\
\hline Giant cell arteritis & 0 & 0 & $4(0.1 \%)$ & $1(0.0 \%)$ \\
\hline Myalgia, myositis, rheumatism & $10(5.0 \%)$ & $1(0.5 \%)$ & $229(4.1 \%)$ & $2(0.0 \%)$ \\
\hline Pain in joints & $45(22.4 \%)$ & $5(2.5 \%)$ & $952(17.2 \%)$ & $20(0.4 \%)$ \\
\hline PMR & 0 & 0 & $11(0.2 \%)$ & $2(0.0 \%)$ \\
\hline Polymyositis & 0 & 0 & $1(0.0 \%)$ & 0 \\
\hline PSO & $5(2.5 \%)$ & $1(0.5 \%)$ & $57(1.0 \%)$ & $2(0.0 \%)$ \\
\hline PsA & $4(2.0 \%)$ & $2(1.0 \%)$ & $8(0.1 \%)$ & $3(0.1 \%)$ \\
\hline RA & $3(1.5 \%)$ & $1(0.5 \%)$ & $44(0.8 \%)$ & $14(0.2 \%)$ \\
\hline Sarcoidosis & 0 & 0 & $12(0.2 \%)$ & 0 \\
\hline Sicca syndrome & $3(1.5 \%)$ & 0 & $9(0.2 \%)$ & $3(0.1 \%)$ \\
\hline Systemic lupus erythematosus & $1(0.5 \%)$ & 0 & $6(0.1 \%)$ & $1(0.0 \%)$ \\
\hline Systemic sclerosis & 0 & 0 & $1(0.0 \%)$ & 0 \\
\hline
\end{tabular}

$A S$ ankylosing spondylitis, $P M R$ polymyalgia rheumatica, $P s A$ psoriatic arthritis, $P S O$ psoriasis, $R A$ rheumatoid arthritis, $r h-$ ir $A E$ rheumatologic immune-related adverse event

an rh-irAE was $0.8 \%(n=43)$, with pain in joints being the most common symptom $(0.4 \%$, $n=20)$.

\section{Patients with a History of a Rheumatic Disease in Baseline}

A total of $201 / 5722$ patients $(3.5 \%)$ that initiated ICI therapy had a history of pre-existing rheumatic disease, defined by either diagnosis for a rheumatologic condition $(n=163)$ or a claim for biologic therapy $(n=39)$ during the 6-month baseline period (Fig. 1). One patient had both a prior rheumatologic diagnosis and a claim for biologic therapy; thus, 162 and 38 patients, respectively, had a claim for selected rheumatologic condition or biologic therapy alone.
Among the 201 patients with a history of rheumatic disease in baseline, $71.6 \%(n=144)$ had $\geq 1 \mathrm{rh}$-irAE in follow-up. The mean time to first rh-irAE was 2.5 months following ICI initiation, and $20.9 \%(n=42)$ of patients had an rh-irAE and a rheumatologist provider on the diagnostic claim (Table 1). When limiting to rhirAEs for which the patient had no diagnoses in baseline, $35.3 \%(n=71)$ had $\geq 1$ rh-irAE, and $5.0 \% \quad(n=10)$ had a new rh-irAE and a rheumatologist provider on the claim (Table 2).

\section{DISCUSSION}

This analysis reports the occurrence of rh-irAEs in patients initiating ICI therapy, thus adding to 


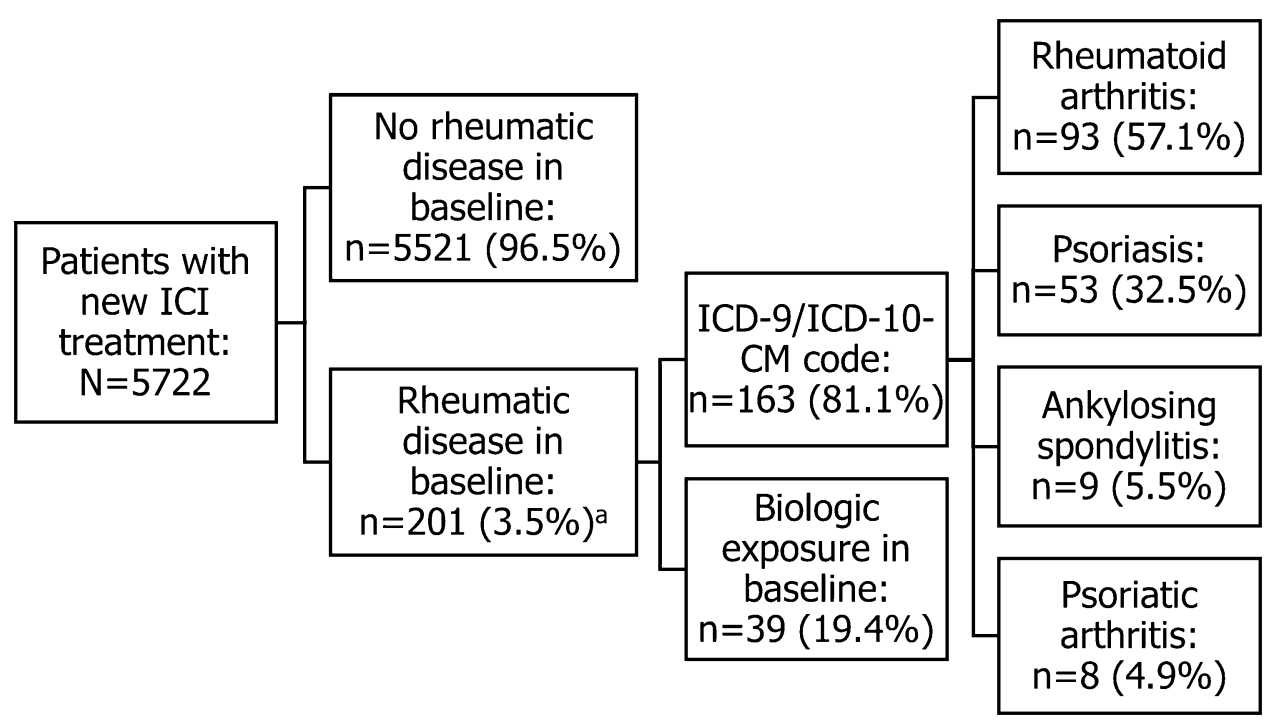

Fig. 1 Patient population within $\operatorname{IBM}^{\circledR}$ MarketScan ${ }^{\circledR}$,

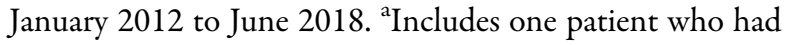
both an ICD-9/ICD-10-CM code and biologic exposure at baseline. ICD-9 or ICD-10-CM International

the current body of knowledge by presenting a broad description of the rh-irAE subtype, which is commonly underreported or absent. This analysis demonstrates that patients with a prior history of rheumatic disease have a higher occurrence of rh-irAEs, as $29.6 \%$ of patients without a history of rheumatic disease experienced an rh-irAE, compared to $71.6 \%$ of patients with a history of rheumatic disease. Historically, patients with a history of rheumatic disease were excluded from clinical trials and safety studies; therefore, this observed increase of rh-irAEs is important for clinical management of patients [15]. Of note, while there are no guidelines that strictly require a pause in treatment with disease-modifying antirheumatic drugs (DMARDs) in patients with rheumatic disease upon initiation of ICI therapy, this may occur in some cases, and therefore could partially explain the variation in rates of rh-irAEs between patients with and without a history of rheumatic disease.

Frequencies of rh-irAEs decreased as the inclusion criteria for diagnostic claims became more stringent (e.g., rheumatologist exposure or events for which there was no baseline diagnosis). This suggests that many patients are
Classification of Diseases 9 or International Classification of Diseases 10-Clinical Modification; ICI immune checkpoint inhibitor

not referred to a rheumatologist, and/or the referral does not result in confirmation of a rheumatologic diagnosis by the rheumatologist. If symptoms were mild and/or managed by the oncologist, a referral may not have been warranted, since guidelines for management of irAEs are based upon the symptom grade severity, and state physician consideration for rheumatology should occur at grade 2 severity. However, a multidisciplinary approach and high suspicion of musculoskeletal symptoms from the physician is needed for early recognition [15].

Due to inconsistent reporting of specific irAEs in publications, heterogeneity of case definitions, and limited generalizability due to the use of single-center data, an accurate comparison of epidemiologic estimates from the literature is difficult. Richter and Crowson [14] performed a retrospective medical record review of 1293 patients treated with ICI therapy at the Mayo Clinic in Rochester, MN, USA, including all cases of rh-irAEs regardless of grade. Four syndromes under the rh-irAE umbrella were explored, defined based on the clinical diagnosis by a managing oncologist or rheumatologist and categorized as inflammatory arthritis 
(required $\geq 1$ synovitis feature), myopathy (required biopsy, electromyography, or elevated creatine kinase level), vasculitis, or connective tissue disease (CTD). Using this list of rheumatologic syndromes and diagnostic criteria, the prevalence of rh-irAEs in this population was $3.3 \%$. The generalizability of these results to a population-based cohort is limited, since these patients were identified within a tertiary care center in a demographically uniform county.

A prospective study assessed safety events at follow-up visits among 524 patients treated with ICIs at a single center in France from September 2015 to May 2017 [10]. All patients with musculoskeletal symptoms were referred to a rheumatologist for evaluation, including those with a history of autoimmune disease if they had a flare or new onset of symptoms. Thirty-five patients were evaluated by a rheumatologist for rh-irAEs; two of these patients had previously been diagnosed with axial spondyloarthritis and psoriatic arthritis. Twenty referred patients had inflammatory arthritis (IA) diagnoses; the other 15 were found to have non-inflammatory musculoskeletal conditions. Thus, 20/524 patients, or $3.8 \%$, experienced a new rh-irAE in follow-up.

The present study bridges the gap in the current body of literature by quantifying the occurrence of a broad range of rh-irAEs in a large population. The overall frequencies of events, the types of events (most were nonspecific joint pain and related diagnoses), and the low rates of exposure to rheumatologists suggest that severe rh-irAEs are uncommon in this population. Nevertheless, awareness of the potential for these events is warranted, particularly among patients with a history of rheumatic disease.

\section{Strengths and Limitations}

This study has limitations that should be acknowledged when interpreting the results. It is not possible to assess any causality between treatment and outcomes in claims analyses. Therefore, these events are potential rh-irAEs, and a physician's assessment would be necessary to judge whether the event was related to
ICI exposure rather than a particular pre-existing disease, if applicable. The most common events observed were not rheumatic diseases but rather symptoms, such as pain in joints. Clinicians note that it is difficult to accurately identify the causes of arthralgia and myalgia in cancer patients; thus, analyses may be augmenting the associated prevalence of rh-irAEs by attributing this diagnosis to ICI treatment rather than the underlying malignancy [5].

Additionally, this analysis is limited by the ability to accurately identify diagnoses within the IBM $^{\circledR}$ MarketScan ${ }^{\circledR}$ database, which relies on accurate diagnostic coding and excludes patients who are not commercially insured, reducing the generalizability of these results. Moreover, the generalizability is also limited to the defined rheumatic disease areas included in this analysis (PSO, PsA, RA, and AS). Patients with a history of rheumatologic conditions diagnosed earlier than the 6-month baseline period would have been misclassified in our stratified analysis. Although this analysis explored all available follow-up time for patients undergoing ICI therapy, some rh-irAEs could have been missed among patients with shorter duration of ICI therapy (less than the required 6 months), and rh-irAEs were not captured if they occurred after discontinuation of ICI therapy.

Furthermore, while some patients were under treatment with biologics in the baseline period, it is unknown whether these patients continued treatment after initiating ICI therapy. This is a limitation of larger database analyses when compared to single-center series, which may allow for tighter control over the collection of these data, whereas retrospective database analyses are restricted to available information. However, single-center series lack the geographic heterogeneity and large sample size of the present analysis. In addition, while patients without evidence of a diagnosis of PSO, PsA, RA, or AS could be classified as having preexisting rheumatic disease due to the presence of a claim for a biologic, it is possible that biologics were prescribed for non-rheumatologic conditions.

Another important limitation is the lack of information about the type of cancer for which 
a given patient is being treated. Clinical trial data have shown that subtypes of irAEs vary by ICI class, however, the low frequencies reported for individual rh-irAEs and organ syndromes restrict the analysis from stratifications and examination of covariates. Additionally, the relatively low frequency of exposure to rheumatologists could be due to differences within institutional referral procedures, rather than severity of the rh-irAEs.

\section{CONCLUSIONS}

The analysis of data from a large, geographically diverse database of patients with generalizability to the commercially insured population in the US shows that overall occurrence of severe rh-irAEs is low and that most rh-irAEs are not specific rheumatic diseases, but rather descriptions of symptoms such as joint pain. While specific and severe rh-irAEs do occur and should receive appropriate rheumatologic referral, these results suggest that other subtypes of irAEs are more common.

\section{ACKNOWLEDGEMENTS}

Funding. Sponsorship for this study and the Rapid Service Fee were funded by UCB Pharma.

Medical Writing, Editorial, and/or Other Assistance. Medical writing and editorial assistance in the preparation of this article was provided by Aaron Keeling, BA, Costello Medical, Boston, MA, USA. The authors would also like to acknowledge Mylene Serna, PharmD, UCB Pharma, Smyrna, GA, USA for publication coordination and Janek Rudnik, PhD, UCB Pharma for analytical support. Support for this assistance was funded by UCB Pharma in accordance with Good Publication Practice (GPP3) guidelines.

Authorship. All named authors meet the International Committee of Medical Journal Editors (ICMJE) criteria for authorship for this article, take responsibility for the integrity of the work as a whole, and have given their approval for this version to be published.

Author Contributions. Substantial contributions to study conception and design: Suzanne Anjohrin, Anna Sheahan, Robert Suruki, Jeffrey L. Stark, Victor S. Sloan; substantial contributions to analysis and interpretation of the data: Suzanne Anjohrin, Anna Sheahan, Robert Suruki, Jeffrey L. Stark, Victor S. Sloan; drafting the article or revising it critically for important intellectual content: Suzanne Anjohrin, Anna Sheahan, Robert Suruki, Jeffrey L. Stark, Victor S. Sloan; final approval of the version of the article to be published: Suzanne Anjohrin, Anna Sheahan, Robert Suruki, Jeffrey L. Stark, Victor S. Sloan.

Disclosures. Suzanne Anjohrin, Anna Sheahan, and Jeffrey L. Stark are employees of and shareholders in UCB Pharma. Robert Suruki is an employee of Janssen Pharmaceuticals, former employee of UCB Pharma, and shareholder in UCB Pharma and GSK. Victor S. Sloan is an employee of the Peace Corps and clinical faculty of Rutgers Robert Wood Johnson Medical School. This article was prepared by Victor Sloan in his personal capacity. The views expressed are his own and do not necessarily reflect the views of the Peace Corps or the United States government.

Compliance with Ethics Guidelines. Formal ethical review boards were not consulted for this study. All analysis was performed via deidentified and anonymized database with permission of the data owner to be performed and presented at an aggregate level. The database is Health Insurance Portability and Accountability Act (HIPAA) compliant, and all patient data were de-identified before delivery. The study was conducted in accordance with the Helsinki Declaration of 1964, and its later amendments.

Data Availability. The datasets generated during and/or analyzed during the current study are not publicly available, as data from non-interventional studies are outside of UCB's data sharing policy. 
Open Access. This article is licensed under a Creative Commons Attribution-NonCommercial 4.0 International License, which permits any non-commercial use, sharing, adaptation, distribution and reproduction in any medium or format, as long as you give appropriate credit to the original author(s) and the source, provide a link to the Creative Commons licence, and indicate if changes were made. The images or other third party material in this article are included in the article's Creative Commons licence, unless indicated otherwise in a credit line to the material. If material is not included in the article's Creative Commons licence and your intended use is not permitted by statutory regulation or exceeds the permitted use, you will need to obtain permission directly from the copyright holder. To view a copy of this licence, visit http://creativecommons.org/licenses/by$\mathrm{nc} / 4.0 /$.

\section{REFERENCES}

1. Darvin P, Toor SM, Sasidharan Nair V, Elkord E. Immune checkpoint inhibitors: recent progress and potential biomarkers. Exp Mol Med. 2018;50(12): $1-11$.

2. Haanen JB, Robert C. Immune checkpoint inhibitors. Prog Tumor Res. 2015;42:55-66.

3. Press release: The Nobel Prize in Physiology or Medicine [press release]. 2018.

4. Suarez-Almazor ME, Kim ST, Abdel-Wahab N, Diab A. Review: immune-related adverse events with use of checkpoint inhibitors for immunotherapy of cancer. Arthritis Rheumatol. 2017;69(4):687-99.

5. Abdel-Rahman O, Eltobgy $\mathrm{M}$, Oweira $\mathrm{H}$, et al. Immune-related musculoskeletal toxicities among cancer patients treated with immune checkpoint inhibitors: a systematic review. Immunotherapy. 2017;9(14):1175-83.

6. Abdel-Wahab N, Shah M, Lopez-Olivo MA, SuarezAlmazor ME. Use of immune checkpoint inhibitors in the treatment of patients with cancer and preexisting autoimmune disease: a systematic review. Ann Intern Med. 2018;168(2):121-30.

7. Benfaremo D, Manfredi L, Luchetti MM, Gabrielli A. Musculoskeletal and rheumatic diseases induced by immune checkpoint inhibitors: a review of the literature. Curr Drug Saf. 2018;13(3):150-64.

8. Gordon R, Kasler MK, Stasi K, et al. Checkpoint inhibitors: common immune-related adverse events and their management. Clin J Oncol Nurs. 2017;21(2 Suppl):45-52.

9. Khoja L, Day D, Wei-Wu Chen T, Siu LL, Hansen AR. Tumour- and class-specific patterns of immunerelated adverse events of immune checkpoint inhibitors: a systematic review. Ann Oncol. 2017;28(10):2377-85.

10. Kostine M, Rouxel L, Barnetche T, et al. Rheumatic disorders associated with immune checkpoint inhibitors in patients with cancer-clinical aspects and relationship with tumour response: a singlecentre prospective cohort study. Ann Rheum Dis. 2018;77(3):393-8.

11. Cappelli LC, Gutierrez AK, Bingham CO 3rd, Shah AA. Rheumatic and musculoskeletal immune-related adverse events due to immune checkpoint inhibitors: a systematic review of the literature. Arthritis Care Res (Hoboken). 2017;69(11):1751-63.

12. El Osta B, Hu F, Sadek R, Chintalapally R, Tang SC. Not all immune-checkpoint inhibitors are created equal: meta-analysis and systematic review of immune-related adverse events in cancer trials. Crit Rev Oncol Hematol. 2017;119:1-12.

13. Lidar M, Giat E, Garelick D, et al. Rheumatic manifestations among cancer patients treated with immune checkpoint inhibitors. Autoimmun Rev. 2018;17(3):284-9.

14. Richter MD, Crowson C, Kottschade LA, et al. Rheumatic syndromes associated with immune checkpoint inhibitors: a single-center cohort of sixty-one patients. Arthritis Rheumatol. 2019;71(3): 468-75.

15. Lee KA, Kim HR, Yoon SY. Rheumatic complications in cancer patients treated with immune checkpoint inhibitors. Korean J Intern Med. 2019;34(6):1197-209. 